Article

www.mdpi.com/journal/materials

\title{
Influence of the Structural Properties of Mesoporous Silica on the Adsorption of Guest Molecules
}

\author{
Hanna Ritter, Jan Hinrich Ramm and Dominik Brühwiler * \\ Institute of Inorganic Chemistry, University of Zurich, Winterthurerstrasse 190, CH-8057 Zurich, \\ Switzerland
}

* Author to whom correspondence should be addressed; E-Mail: bruehwi@aci.uzh.ch; Tel.: +41 44635 4630; Fax: +41446356802.

Received: 11 August 2010; in revised form: 18 August 2010 / Accepted: 18 August 2010 / Published: 25 August 2010

\begin{abstract}
Amino-functionalized mesoporous silica of different pore sizes and pore system dimensionalities is used as a host material for the inclusion of fluorescein (non-covalent host-guest interaction) and fluorescein isothiocyanate (covalent host-guest interaction). The parameters determining the achievable guest loading depend on the type of host-guest interaction. For covalent interaction, the loading is mainly determined by the accessibility of the adsorption sites, while a more complex situation was encountered in case of noncovalent interactions. In addition to the accessibility of the adsorption sites, an interpretation of the results needs to take into account the confinement of the included guests, as well as the distribution of the adsorption sites.
\end{abstract}

Keywords: mesoporous silica; host-guest; adsorption; interaction; amine; fluorescein; FITC

\section{Introduction}

The introduction of guests into nanoporous hosts with defined pore sizes is a versatile concept for producing materials with new properties and promising possibilities for various applications [1-4]. Examples include photonic antenna systems (host $=$ zeolite L, guest $=$ luminescent molecules) [4], drug delivery devices (host $=$ mesoporous silica, guest $=$ drug molecules) [5-7], and biocatalysts (host $=$ mesoporous silica, guest $=$ enzymes) [8]. The discovery of mesoporous silica with ordered 
pores $[9,10]$ has extended this field by allowing the incorporation of large guest species such as DNA [11], preformed quantum dots [12], or semiconducting polymers [13]. When preparing host-guest materials of this kind, questions regarding the ideal pore size and pore system arise. Using a model consisting of fluorescein and fluorescein isothiocyanate (FITC) as guests and various amino-functionalized mesoporous silica materials as hosts, we illustrate that the answer to these questions very much depends on the particular host-guest interaction.

The pore size of a host material determines the accessibility of the adsorption sites. It is intuitively immediately clear that the accessibility increases with increasing pore size, thereby enabling the preparation of highly loaded host-guest materials. However, large pore sizes usually promote leaching, particularly in cases where the interaction between guest and host is weak. This leads to experimental problems, because the preparation of host-guest systems based on nanoporous materials usually includes washing steps to remove guest species adsorbed on the external particle surface. In case of a non-covalent interaction between guest and host, it is therefore necessary to optimize the accessibility of the adsorption sites without compromising the confinement. Confinement can be a crucial factor in determining the stability of host-guest systems, as shown by the inclusion of organic molecules into the narrow channels of zeolite L, where it has been observed that leaching can be suppressed under certain conditions, even when washing with a solvent in which the guests are well soluble [14].

When working with covalent host-guest interactions, leaching of the guests becomes much less of a problem. However, in terms of the synthesis of the respective host-guest systems, pore blocking effects by irreversible binding of guests to pore entrance sites have to be taken into account when choosing an appropriate host material.

\section{Results and Discussion}

\subsection{Mesoporous Silica}

To study the effect of the pore size on the ability of the host materials to accommodate fluorescein and FITC, we have employed various mesoporous silicas. The key characteristics of the parent materials (before amino-functionalization), namely the pore diameter $\left(\mathrm{d}_{\mathrm{BJH}}\right)$, the BET surface area $\left(\mathrm{S}_{\mathrm{BET}}\right)$, the external particle surface area $\left(\mathrm{S}_{\mathrm{Ext}}\right)$, the total pore volume $\left(\mathrm{V}_{\mathrm{tot}}\right)$, and the primary mesopore volume $\left(\mathrm{V}_{\mathrm{P}}\right)$, are summarized in Table 1. Pore size distributions are given in Figure 1. It should be noted that the $\mathrm{BJH}$ method tends to underestimate the absolute pore size [15]. This is illustrated by comparison with values obtained using the geometrical method proposed by Kruk et al., which additionally takes into account the X-ray diffraction data and yields reliable results for well defined MCM-41 type systems [16]. Applying this method resulted in a pore diameter of $2.89 \mathrm{~nm}$ for MCM-41(s) and $3.76 \mathrm{~nm}$ for MCM-41. All materials feature particles of irregular morphology with particle sizes in the range of $1-2 \mu \mathrm{m}$.

Apart from the pore sizes, there are further fundamental differences between the materials. While MCM-41(s), MCM-41, and SBA-15 have one-dimensional channel systems (hexagonal), the pore system of MCM-48 is three-dimensional (cubic) (Figure 2) [10]. SBA-15, on the other hand, has a property that is commonly not found in materials of the MCM-41 and MCM-48 type. The pore walls of SBA-15 contain micropores, depending to a certain extent on the synthesis conditions [17]. In our case, a micropore volume of $0.09 \mathrm{~cm}^{3} / \mathrm{g}$ was found. 
Table 1. Properties of the parent mesoporous silica materials.

\begin{tabular}{lccccc}
\hline & $\mathbf{d}_{\text {BJH }}[\mathbf{n m}]$ & $\mathbf{S}_{\text {BET }}\left[\mathbf{m}^{2} / \mathbf{g}\right]$ & $\mathbf{S}_{\text {Ext }}\left[\mathbf{m}^{2} / \mathbf{g}\right]$ & $\mathbf{V}_{\text {tot }}\left[\mathbf{c m}^{3} / \mathbf{g}\right]$ & $\mathbf{V}_{\mathbf{P}}\left[\mathbf{c m}^{3} / \mathbf{g}\right]$ \\
\hline MCM-41(s) & 1.96 & 776 & 35 & 0.48 & 0.44 \\
MCM-48 & 2.52 & 1320 & 173 & 0.98 & 0.86 \\
MCM-41 & 2.82 & 872 & 66 & 0.74 & 0.67 \\
SBA-15 & 6.46 & 908 & 74 & 1.21 & 1.09 \\
\hline
\end{tabular}

Amino-functionalized materials were obtained by the well established reaction with 3-aminopropyltrimethoxysilane (APTMS). Samples with low (approximately $0.45 \mu \mathrm{mol} / \mathrm{m}^{2}$ ) and high amino content (approximately $1.35 \mu \mathrm{mol} / \mathrm{m}^{2}$ ) were prepared by adding the corresponding amounts of APTMS to a suspension of the mesoporous silica in dry toluene. Indeed, analysis of the amino-functionalized samples revealed that grafting of APTMS was close to quantitative under our conditions, and led, as expected, to a reduction of the average pore diameter, pore volume, and BET surface area of the materials. The respective pore size distributions are shown in Figure 1.

Figure 1. Pore size distributions (BJH, desorption isotherm) of parent and amino-functionalized mesoporous silicas.

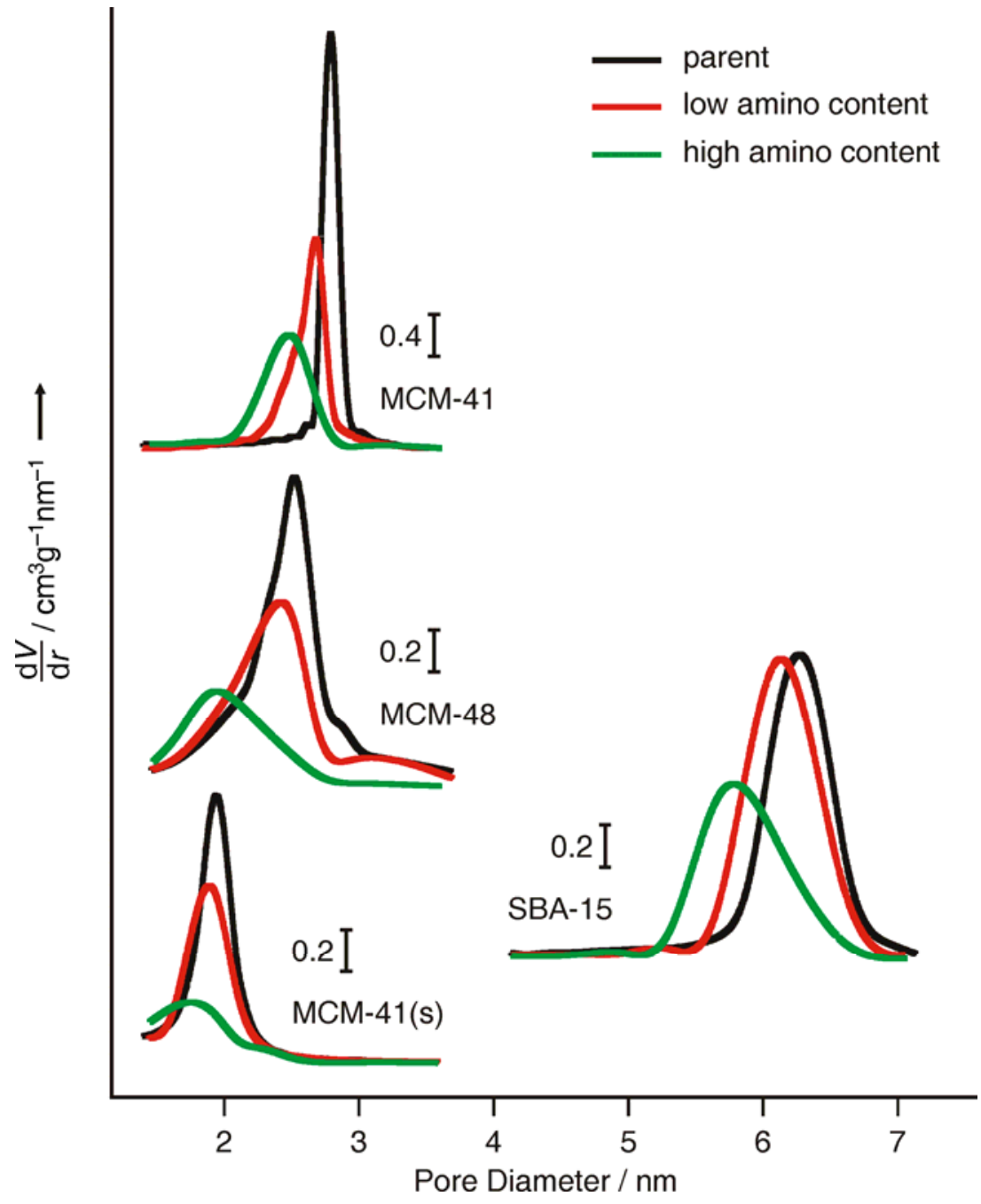


Figure 2. X-ray diffraction patterns of parent MCM-41(s) (left panel), MCM-41(middle panel), and MCM-48(right panel).
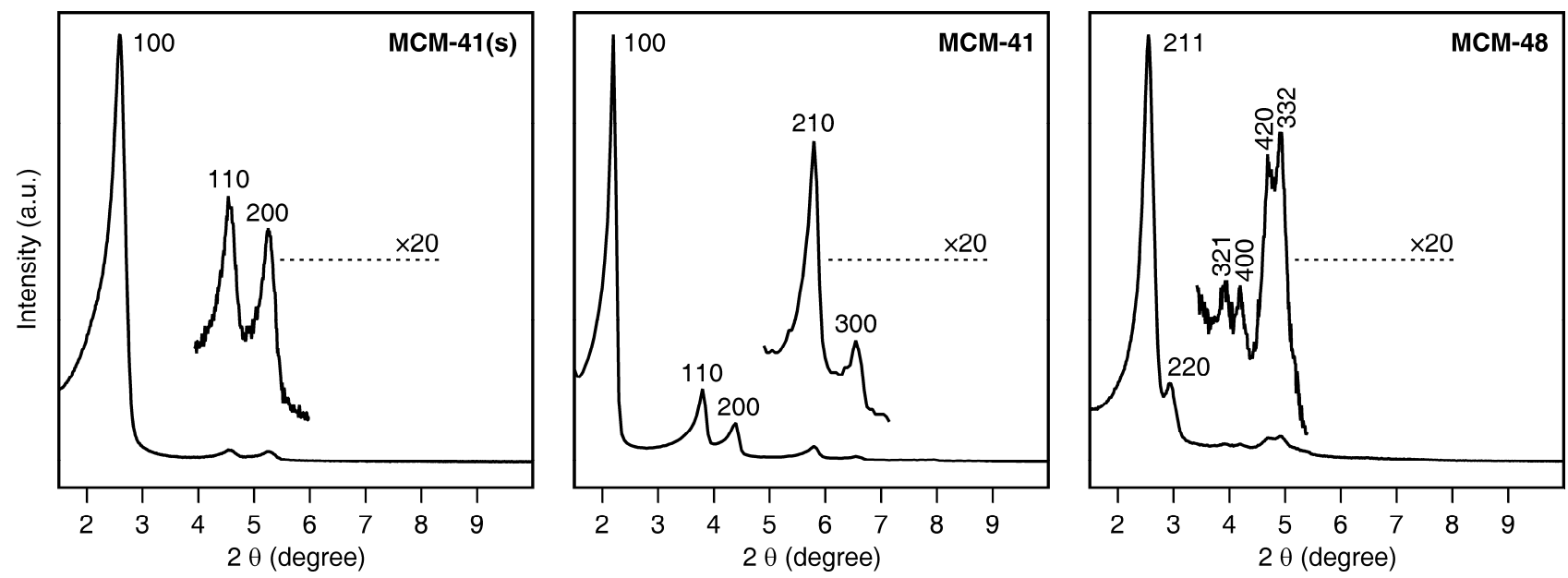

\subsection{Loading with FITC and Fluorescein}

FITC is a popular amine labeling reagent, forming a robust thiourea upon reaction. In combination with mesoporous silica, FITC has been used to visualize the distribution of surface-grafted amino groups [18-20], as well as to prepare materials with $\mathrm{pH}$ sensing [21] and bioimaging capabilities [22-25]. Independent loading experiments with the same amino-functionalized sample gave FITC contents that were typically within $\pm 10 \%$. The interpretation of the FITC loading experiments (Figure 3, left) is rather straightforward and can be reduced to an assessment of the accessibility of the amino groups [26]. Having the smallest pore size, MCM-41(s) is able to bind only small amounts of FITC. This is most likely due to pore blocking upon reaction of FITC with amino groups located close to the pore entrances (Figure 4). It should be noted in this context that the distribution of the grafted amino groups is most likely non-uniform, especially in materials with small mesopores, leading to higher grafting densities near the pore entrances [27-29], and thus further promoting pore blocking upon reaction with FITC. Such effects are less pronounced in MCM-41 as a consequence of the larger pores, leading to increased amounts of FITC-labeled amino groups. The relative amount of coupled FITC decreases upon increasing the amino content, because the probability of FITC binding to pore entrance sites increases (Figure 4). Given the pore size of MCM-48, it is very likely that pore blocking occurs in a similar manner as observed for MCM-41. Due to its three-dimensional channel system, the effect of pore blocking on the accessibility of the amino groups, and therefore on the amount of coupled FITC, is, however, less dramatic. Following this line of reasoning, one would expect to find the largest amounts of bound FITC in the amino-functionalized SBA-15. While this is indeed the case for high amino loading, the SBA-15 sample containing small amounts of grafted amino groups gave a comparatively low amount of coupled FITC. This is in agreement with a previous study that led to the conclusion that APTMS grafts preferentially to the intrawall micropores, thereby becoming unavailable for reaction with FITC [26]. 
Figure 3. Amount of included FITC (left) and fluorescein (right) relative to the amount of surface-grafted amino groups for low (hatched bars) and high (black bars) amino contents.
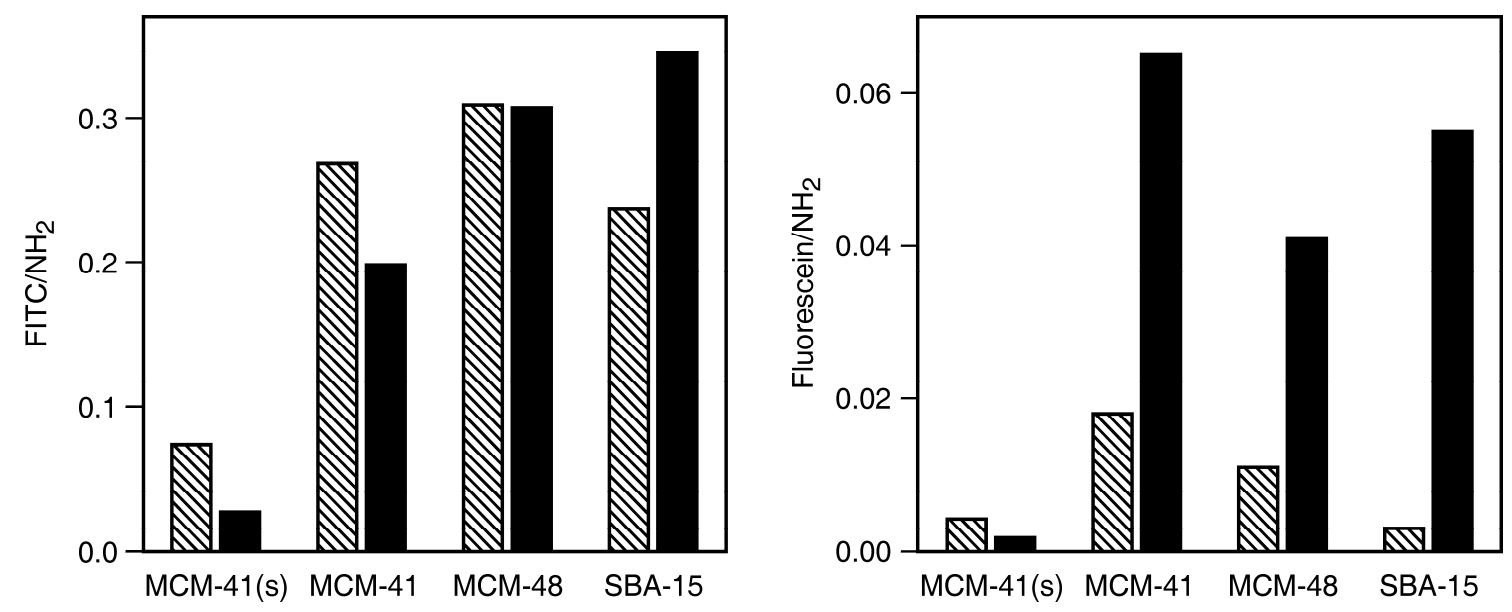

Figure 4. Schematic snapshots illustrating the reaction of FITC with amino-functionalized MCM-41(s) and MCM-41.
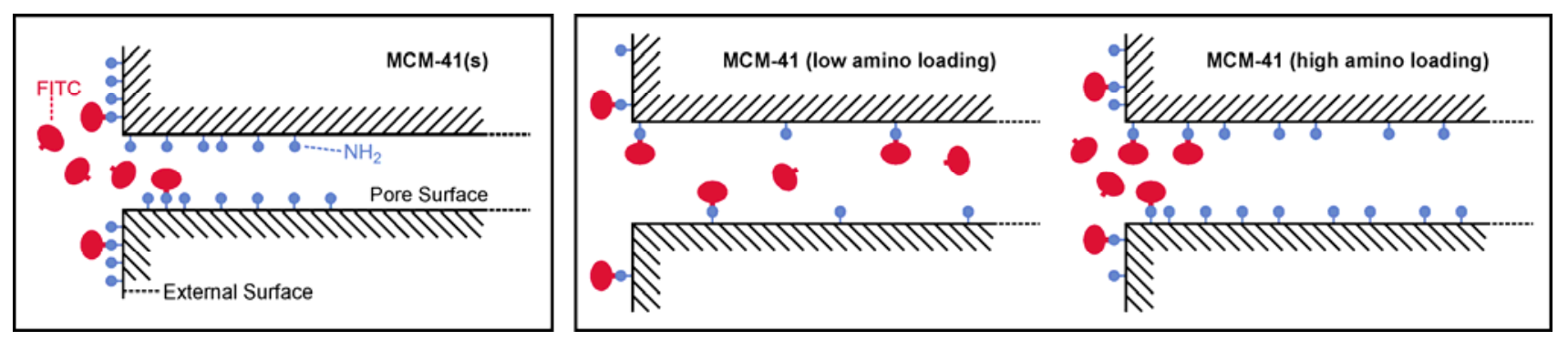

In the case of fluorescein, the results of the loading experiments (Figure 3, right) and their interpretation are more complex due to the reversible interaction of the guest molecules with the host. As expected, the amounts of included fluorescein are much smaller compared to the amounts of bound FITC. Nonetheless, we found the results to be well reproducible. Independent loading experiments with the same amino-functionalized sample gave fluorescein contents that were within $\pm 10 \%$.

With $\mathrm{pK}_{\mathrm{a}}$ values of 4.4 (neutral fluorescein) and 6.7 (fluorescein monoanion) [30], we can expect protonation of surface-grafted amino groups and subsequent electrostatic interaction with fluorescein mono- and dianions. Fluorescein contents clearly show a dependence on the pore size that is different to the one observed for FITC, thus indicating that in addition to the accessibility of the amino groups, there must be further parameters determining the amount of adsorbed fluorescein. MCM-41 in particular is able to retain an uncharacteristically large amount of fluorescein. This can be explained on the basis that fluorescein molecules adsorbed in the channels are well protected against washing with ethanol, whereas in the case of MCM-41(s) most of the fluorescein molecules are located on or close to the exposed external surface. Similarly, a large pore diameter (SBA-15) leads to less confinement, rendering the adsorbed fluorescein molecules susceptible to removal by washing. In this context, it is interesting to note that the dependence of the leaching rate on the pore diameter of a host can potentially be used to control the release of substances [31]. Regarding the microporosity of SBA-15, we can conclude that based on the amount of retained fluorescein, adsorption in the intrawall 
micropores, which would provide strong confinement, is unlikely. Again it is instructive to compare the performances of MCM-41 and MCM-48. The three-dimensional channel system apparently facilitates the removal of adsorbed fluorescein molecules during the washing process.

In contrast to the loading experiments with FITC, the amounts of included fluorescein (relative to the amount of amino groups) are considerably higher in case of high amino contents. To explain this significant difference between low and high amino contents, we have to consider that site-isolated amino groups are more likely at low amino contents. While such site-isolated amino groups can effectively bind FITC, they are less capable of adsorbing and retaining fluorescein. At high amino contents, on the other hand, one can expect a certain degree of APTMS cross-linking, leading to surface-anchored clusters of aminopropyl moieties [32]. Such patches of closely spaced amino groups can effectively adsorb and retain fluorescein.

\section{Experimental Section}

\subsection{Synthesis of Mesoporous Silica}

MCM-41 and MCM-41(s) [26]: $2.20 \mathrm{~g}$ of hexadecyltrimethylammonium bromide (CTAB, Fluka) was dissolved under slight warming (approx. $35{ }^{\circ} \mathrm{C}$ ) in a mixture of $52 \mathrm{~mL}$ of $\mathrm{H}_{2} \mathrm{O}$ and $24 \mathrm{~mL}$ of aqueous ammonia (28\%, Fluka). An amount of $10 \mathrm{~mL}$ of tetraethoxysilane (TEOS, Fluka) was slowly added under stirring and the resulting gel was further stirred for $3 \mathrm{~h}$ at room temperature. The mixture was transferred to a Teflon-lined autoclave and heated at $110^{\circ} \mathrm{C}$ for $48 \mathrm{~h}$. The product was obtained by filtration, washed with at least $800 \mathrm{~mL}$ of $\mathrm{H}_{2} \mathrm{O}$ and dried overnight in air at room temperature. The structure directing agent (SDA) was removed by first heating at $300{ }^{\circ} \mathrm{C}$ for $2 \mathrm{~h}$ and subsequent calcination in air at $550{ }^{\circ} \mathrm{C}$ for $16 \mathrm{~h}$. Heating rates of $2{ }^{\circ} \mathrm{C} / \mathrm{min}$ were applied.

MCM-41 featuring a smaller pore diameter (MCM-41(s)) was prepared accordingly, using $1.86 \mathrm{~g}$ of dodecyltrimethylammonium bromide (Fluka) instead of CTAB.

MCM-48 [33]: An amount of $8.80 \mathrm{~g}$ of CTAB was dissolved under slight warming (approx. $35{ }^{\circ} \mathrm{C}$ ) in $80 \mathrm{~mL}$ of $\mathrm{H}_{2} \mathrm{O}$. After the addition of $10 \mathrm{~mL}$ of $2 \mathrm{M}$ aqueous $\mathrm{NaOH}, 10 \mathrm{~mL}$ of TEOS was added dropwise under stirring. After further stirring for $30 \mathrm{~min}$, the mixture was transferred to a Teflon-lined autoclave and heated at $100{ }^{\circ} \mathrm{C}$ for $72 \mathrm{~h}$. The product was recovered by filtration, washed with at least $1 \mathrm{~L}$ of $\mathrm{H}_{2} \mathrm{O}$ and oven-dried overnight at $80{ }^{\circ} \mathrm{C}$. The SDA was removed by first heating at $300{ }^{\circ} \mathrm{C}$ for $2 \mathrm{~h}$ and subsequent calcination in air at $550{ }^{\circ} \mathrm{C}$ for $8 \mathrm{~h}$. Heating rates of $2{ }^{\circ} \mathrm{C} / \mathrm{min}$ were applied.

SBA-15 [34]: $2.20 \mathrm{~g}$ of Pluronic P123 $\left(\mathrm{EO}_{20} \mathrm{PO}_{70} \mathrm{EO}_{20}, \mathrm{M}_{\mathrm{av}}=5800\right.$, Aldrich) was dissolved in a mixture of $49 \mathrm{~mL}$ of $\mathrm{H}_{2} \mathrm{O}$ and $31 \mathrm{~mL}$ of $4 \mathrm{M}$ aqueous $\mathrm{HCl}$. To this clear solution, $5 \mathrm{~mL}$ of TEOS was slowly added under stirring. After further stirring for $20 \mathrm{~h}$ at approximately $35^{\circ} \mathrm{C}$, the mixture was transferred to a Teflon-lined autoclave and heated at $100{ }^{\circ} \mathrm{C}$ for $24 \mathrm{~h}$. The product was obtained by filtration and washed with at least $1 \mathrm{~L}$ of $\mathrm{H}_{2} \mathrm{O}$. After drying the material overnight in air at room temperature, the SDA was removed by heating in air at $500{ }^{\circ} \mathrm{C}$ for $16 \mathrm{~h}$, with a heating rate of $1{ }^{\circ} \mathrm{C} / \mathrm{min}$. 


\subsection{Reaction with APTMS}

For the functionalization with 3-aminopropyltrimethoxysilane (APTMS, Fluka), $500 \mathrm{mg}$ of calcined mesoporous silica was dispersed in $30 \mathrm{~mL}$ of dry toluene (Fluka, puriss.). After the addition of a calculated amount of APTMS (taking into account the surface area of the employed mesoporous silica and a quantitative grafting yield), the suspension was refluxed for $3 \mathrm{~h}$. The functionalized product was recovered by filtration, washed with $100 \mathrm{~mL}$ of ethanol, and cured at $80{ }^{\circ} \mathrm{C}$ for $1 \mathrm{~h}$.

\subsection{Loading with FITC and Fluorescein}

A calculated amount (1.5-fold excess relative to the amount of grafted amino groups) of FITC (fluorescein 5-isothiocyanate, Fluka) or fluorescein (free acid, Riedel-de Haën) was dissolved in $25 \mathrm{~mL}$ of absolute ethanol. After the addition of $250 \mathrm{mg}$ of amino-functionalized mesoporous silica, the suspension was stirred for $24 \mathrm{~h}$ at room temperature. The yellow product was recovered by filtration and washed with $50 \mathrm{~mL}$ of ethanol. After redispersion in $50 \mathrm{~mL}$ of fresh ethanol and stirring for $15 \mathrm{~min}$, the final product was recovered by filtration, washed with $50 \mathrm{~mL}$ of ethanol and oven-dried at $80{ }^{\circ} \mathrm{C}$ for $1 \mathrm{~h}$.

The amount of included FITC or fluorescein was determined by dissolving the sample in $25 \mathrm{~mL}$ of $0.2 \mathrm{M}$ aqueous $\mathrm{NaOH}$ and measuring the UV-Vis absorption spectrum of the resulting clear solution after appropriate dilution. Repeated analysis of the same sample gave an average relative error of $3 \%$. An extinction coefficient of $\varepsilon=75^{\prime} 000 \mathrm{M}^{-1} \mathrm{~cm}^{-1}$ at $\lambda=490 \mathrm{~nm}$ was used for calculating the concentration [26].

\subsection{Amino Group Analysis}

An amount of $15 \mathrm{mg}$ of amino-functionalized mesoporous silica was stirred in $30 \mathrm{~mL}$ of $0.02 \mathrm{M}$ aqueous $\mathrm{NaOH}$ until completely dissolved. A $100 \mu \mathrm{L}$ aliquot of this solution was transferred into a cuvette $(\mathrm{d}=1 \mathrm{~cm})$ and $2 \mathrm{~mL}$ of phosphate buffer $(0.2 \mathrm{M}, \mathrm{pH} 8.0)$ was added. After the addition of $1 \mathrm{~mL}$ of fluorescamine solution (Sigma, $1 \mathrm{mM}$ in acetone), the fluorescence spectrum was measured by excitation at $366 \mathrm{~nm}$. The emission intensity at $480 \mathrm{~nm}$ was taken as a data point. A calibration line was prepared accordingly by using $100 \mu \mathrm{L}$ aliquots of differently concentrated solutions of APTMS in $30 \mathrm{~mL}$ of $0.02 \mathrm{M}$ aqueous $\mathrm{NaOH}$ (containing $15 \mathrm{mg}$ of the respective dissolved parent silica) [35]. Repeated analysis of the same sample gave an average relative error of $8 \%$.

\subsection{Physical Measurements}

Nitrogen sorption isotherms were collected at $77 \mathrm{~K}$ using a Quantachrome NOVA 2200. Samples were vacuum-degassed at $80{ }^{\circ} \mathrm{C}$ for $3 \mathrm{~h}$. The total surface area $\mathrm{S}_{\mathrm{BET}}$ was calculated by the BET method [36], whereas the external surface area $S_{E x t}$ and the primary mesopore volume $V_{P}$ were determined from the high-pressure linear part of the $\alpha_{S}$-plot [37]. Pore size distributions were calculated from the desorption branches of the nitrogen isotherms using the $\mathrm{BJH}$ method [38]. The total pore volume $\mathrm{V}_{\text {tot }}$ was estimated from the amount of nitrogen adsorbed at a relative pressure of 0.95. A Perkin-Elmer LS50B spectrofluorometer was used for the fluorescamine assays and UV-Vis spectra were measured 
with a Cary 1E spectrophotometer. Powder diffraction patterns were collected on a STOE StadiP diffractometer operating with monochromatized $\mathrm{Cu} \mathrm{K} \alpha_{1}$ radiation.

\section{Conclusions}

The influence of the structural properties of mesoporous silica hosts on the adsorption of guest molecules strongly depends on the specific host-guest interactions. In the case of covalent interactions, the amount of adsorbed molecules is mainly determined by the accessibility of the adsorption sites. Host materials with large pore sizes and multi-dimensional channel systems are advantageous for maximizing the amount of adsorbed guests.

In the case of non-covalent interactions, the spacing or density of the adsorption sites, as well as confinement effects have to be taken into account in addition to the accessibility. Finding a balance between the accessibility of the adsorption sites and confinement is crucial for the synthesis of hostguest systems based on non-covalent interactions. A high accessibility facilitates the inclusion of guest species, but the subsequent lack of confinement causes high leaching rates. Low accessibility can similarly lead to high leaching rates, as guest molecules are preferentially adsorbed at sites on the external surface and on the pore surface close to the pore entrances.

\section{Acknowledgements}

Financial support was provided by the European Commission through the Human Potential Programme (Marie-Curie RTN Nanomatch, Grant No. MRTN-CT-2006-035884) and by the Swiss National Science Foundation (Project 200020-117591).

\section{References and Notes}

1. Moller, K.; Bein, T. Inclusion chemistry in periodic mesoporous hosts. Chem. Mater. 1998, 10, 2950-2963.

2. Scott, B.J.; Wirnsberger, G.; Stucky, G.D. Mesoporous and mesostructured materials for optical applications. Chem. Mater. 2001, 13, 3140-3150.

3. Schulz-Ekloff, G.; Wöhrle, D.; van Duffel, B.; Schoonheydt, R.A. Chromophores in porous silicas and minerals: preparation and optical properties. Microporous Mesoporous Mater. 2002, 51, 91-138.

4. Brühwiler, D.; Calzaferri, G.; Torres, T.; Ramm, J.H.; Gartmann, N.; Dieu, L.-Q.; López-Duarte, I.; Martínez-Díaz, M.V. Nanochannels for supramolecular organization of luminescent guests. J. Mater. Chem. 2009, 19, 8040-8067.

5. Vallet-Regí, M.; Balas, F.; Arcos, D. Mesoporous materials for drug delivery. Angew. Chem. Int. Ed. 2007, 46, 7548-7558.

6. Slowing, I.I.; Vivero-Escoto, J.L.; Wu, C.-W.; Lin, V.S.-Y. Mesoporous silica nanoparticles as controlled release drug delivery and gene transfection carriers. Adv. Drug Delivery Rev. 2008, 60, 1278-1288.

7. Manzano, M.; Vallet-Regí, M. New developments in ordered mesoporous materials for drug delivery. J. Mater. Chem. 2010, 20, 5593-5604. 
8. Hartmann, M. Ordered mesoporous materials for bioadsorption and biocatalysis. Chem. Mater. 2005, 17, 4577-4593.

9. Yanagisawa, T.; Shimizu, T.; Kuroda, K.; Kato, C. The preparation of alkyltrimethy lammonium-kanemite complexes and their conversion to microporous materials. Bull. Chem. Soc. Jpn. 1990, 63, 988-992.

10. Kresge, C.T.; Leonowicz, M.E.; Roth, W.J.; Vartuli, J.C.; Beck, J.S. Ordered mesoporous molecular sieves synthesized by a liquid-crystal template mechanism. Nature 1992, 359, 710-712.

11. Gao, F.; Botella, P.; Corma, A.; Blesa, J.; Dong, L. Monodispersed mesoporous silica nanoparticles with very large pores for enhanced adsorption and release of DNA. J. Phys. Chem. B 2009, 113, 1796-1804.

12. Gao, X.; Nie, S. Doping mesoporous materials with multicolor quantum dots. J. Phys. Chem. B 2003, 107, 11575-11578.

13. Molenkamp, W.C.; Watanabe, M.; Miyata, H.; Tolbert, S.H. Highly polarized luminescence from optical quality films of a semiconducting polymer aligned within oriented mesoporous silica. J. Am. Chem. Soc. 2004, 126, 4476-4477.

14. Brühwiler, D.; Gfeller, N.; Calzaferri, G. Resorufin in the channels of zeolite L. J. Phys. Chem. B 1998, 102, 2923-2929.

15. Ravikovitch, P.I.; Neimark, A.V. Characterization of nanoporous materials from adsorption and desorption isotherms. Colloids Surf. A: Physicochem. Eng. Aspects 2001, 187-188, 11-21.

16. Kruk, M.; Jaroniec, M.; Sayari, A. Adsorption study of surface and structural properties of MCM-41 materials of different pore sizes. J. Phys. Chem. B 1997, 101, 583-589.

17. Galarneau, A.; Cambon, H.; Di Renzo, F.; Fajula, F. True microporosity and surface area of mesoporous SBA-15 as a function of synthesis temperature. Langmuir 2001, 17, 8328-8335.

18. Gartmann, N.; Brühwiler, D. Controlling and imaging the functional-group distribution on mesoporous silica. Angew. Chem. Int. Ed. 2009, 48, 6354-6356.

19. Gartmann, N.; Schütze, C.; Ritter, H.; Brühwiler, D. The effect of water on the functionalization of mesoporous silica with 3-aminopropyltriethoxysilane. J. Phys. Chem. Lett. 2010, 1, 379-382.

20. Ramm, J.H.; Gartmann, N.; Brühwiler, D. Direct synthesis and fluorescent imaging of bifunctionalized mesoporous iodopropyl-silica. J. Colloid Interf. Sci. 2010, 345, 200-205.

21. Wirnsberger, G.; Scott, B.J.; Stucky, G.D. pH Sensing with mesoporous thin films. Chem. Commun. 2001, 119-120.

22. Lin, Y.-S.; Tsai, C.-P.; Huang, H.-Y.; Kuo, C.-T.; Hung, Y.; Huang, D.-M.; Chen, Y.-C.; Mou, C.-Y. Well-ordered mesoporous silica nanoparticles as cell markers. Chem. Mater. 2005, 17, 4570-4573.

23. Hsiao, J.-K.; Tsai, C.-P.; Chung, T.-H.; Hung, Y.; Yao, M.; Liu, H.-M.; Mou, C.-Y.; Yang, C.-S.; Chen, Y.-C.; Huang, D.-M. Mesoporous silica nanoparticles as a delivery system of gadolinium for effective human stem cell tracking. Small 2008, 4, 1445-1452.

24. Tsai, C.-P.; Chen, C.-Y.; Hung, Y.; Chang, F.-H.; Mou, C.-Y. Monoclonal antibody-functionalized mesoporous silica nanoparticles (MSN) for selective targeting breast cancer cells. J. Mater. Chem. 2009, 19, 5737-5743.

25. Lin, Y.-S.; Haynes, C.L. Synthesis and characterization of biocompatible and size-tunable multifunctional porous silica nanoparticles. Chem. Mater. 2009, 21, 3979-3986. 
26. Ritter, H.; Brühwiler, D. Accessibility of amino groups in postsynthetically modified mesoporous silica. J. Phys. Chem. C 2009, 113, 10667-10674.

27. Miyajima, T.; Abry, S.; Zhou, W.; Albela, B.; Bonneviot, L.; Oumi, Y.; Sano, T.; Yoshitake, H. Estimation of spacing between 3-bromopropyl functions grafted on mesoporous silica surfaces by a substitution reaction using diamine probe molecules. J. Mater. Chem. 2007, 17, 3901-3909.

28. Yoshitake, H. Design of functionalization and structural analysis of organically-modified siliceous oxides with periodic structures for the development of sorbents for hazardous substances. J. Mater. Chem. 2010, 20, 4537-4550.

29. Brühwiler, D. Postsynthetic functionalization of mesoporous silica. Nanoscale 2010, 2, 887-892.

30. Martin, M.M.; Lindqvist, L. The pH dependence of fluorescein fluorescence. J. Lumin. 1975, 10, 381-390.

31. Horcajada, P.; Rámila, A.; Pérez-Pariente, J.; Vallet-Regí, M. Influence of pore size of MCM-41 matrices on drug delivery rate. Microporous Mesoporous Mater. 2004, 68, 105-109.

32. Kanan, S.M.; Tze, W.T.Y.; Tripp, C.P. Method to double the surface concentration and control the orientation of adsorbed (3-aminopropyl)dimethylethoxysilane on silica powders and glass slides. Langmuir 2002, 18, 6623-6627.

33. Xu, J.; Luan, Z.; He, H.; Zhou, W.; Kevan, L. A reliable synthesis of cubic mesoporous MCM-48 molecular sieve. Chem. Mater. 1998, 10, 3690-3698.

34. Zhao, D.; Huo, Q.; Feng, J.; Chmelka, B.F.; Stucky, G.D. Nonionic triblock and star diblock copolymer and oligomeric surfactant syntheses of highly ordered, hydrothermally stable, mesoporous silica structures. J. Am. Chem. Soc. 1998, 120, 6024-6036.

35. Ritter, H.; Nieminen, M.; Karppinen, M.; Brühwiler, D. A comparative study of the functionalization of mesoporous silica MCM-41 by deposition of 3-aminopropyltrimethoxysilane from toluene and from the vapor phase. Microporous Mesoporous Mater. 2009, 121, 79-83.

36. Brunauer, S.; Emmett, P.H.; Teller, E. Adsorption of gases in multimolecular layers. J. Am. Chem. Soc. 1938, 60, 309-319.

37. Kruk, M.; Jaroniec, M.; Ryoo, R.; Kim, J.M. Monitoring of the structure of siliceous mesoporous molecular sieves tailored using different synthesis conditions. Microporous Mater. 1997, 12, 93-106.

38. Barrett, E.P.; Joyner, L.G.; Halenda, P.P. The determination of pore volume and area distributions in porous substances. I. Computations from nitrogen isotherms. J. Am. Chem. Soc. 1951, 73, 373-380.

(C) 2010 by the authors; licensee MDPI, Basel, Switzerland. This article is an open access article distributed under the terms and conditions of the Creative Commons Attribution license (http://creativecommons.org/licenses/by/3.0/). 